\title{
Post Liver Transplantation Diarrhea: Etiology, Evaluation and Treatment
}

\author{
Richa Sinha' ${ }^{1}$, Rahul ${ }^{2, *}$
}

Richa Sinha', Rahul2,*

'Department of Microbiology, Indira Gandhi Institute of Medical Sciences, Patna, Bihar, INDIA.

${ }^{2}$ Department of Surgical

Gastroenterology, Sanjay Gandhi Institute of Medical Sciences, Lucknow, Uttar Pradesh, INDIA.

\section{Correspondence}

\section{Dr. Rahul}

Assistant Professor, Department of Surgical Gastroenterology, C- Block, SGPGI (MS), Lucknow-226014, Uttar Pradesh, INDIA.

Mobile no: +91-9565800123

Email: rahulimsbhu@gmail.com

History

- Submission Date: 12-04-2021;

- Revised Date: 11-05-2021;

- Accepted Date: 21-06-2021;

DOI : 10.5530/ijmedph.2021.3.23

Article Available online

http://www.ijmedph.org/v11/i3

Copyright

(C) 2021 Phcog.Net. This is an openaccess article distributed under the terms of the Creative Commons Attribution 4.0 International license.

\begin{abstract}
Diarrhea is a frequent post-operative complication following liver transplantation. It occurs frequently in the initial 3-4 months. Common etiologic factors include immunosuppressive drugs (Mycophenolate mofetil, Tacrolimus and Cyclosporine) and infection (Clostridium difficile, Cytomegalovirus and Rotavirus infection). Inflammatory bowel disease, malignancy and graft versus host disease are infrequent but important causes. Actual incidence of Diarrhea among liver transplant recipients remains elusive as it is often under-reported. However, as per the current literature, it occurs in 10-40 percent of recipients. It can result in dehydration, dyselectrolytemia, derangement of renal profile and even graft loss. The alteration in immunosuppressive drugs in early post-operative period may predispose graft rejection. High index of suspicion and systematic evaluation is pertinent for timely diagnosis. The review summarizes the important causes, epidemiology, recent updates, evaluation and management of the of post liver transplant diarrhea.

Key words: Diarrhea, Liver transplantation, Immunosuppression, Rejection, Infection.
\end{abstract}

\section{INTRODUCTION}

Potent immunosuppressive drugs have bestowed great success to liver transplant (LT). The choice of drugs is based on their efficacy and safety profile. Nearly all of them are associated with gastrointestinal (GI) toxicities. Diarrhea is one of the most frequent complaint post LT and contributes to significant morbidity and mortality in recipients. ${ }^{1}$ It is often associated with dehydration, dyselectrolytemia and rise in creatinine. Chronic diarrhea (lasts $>2$ weeks) may result in weight loss, failure to thrive and fluctuation in the pharmacokinetics of immunosuppressive agents. True incidence of diarrhea remains elusive. It is often under-reported. It ranges from $10 \%$ to $40 \%$ among the solid organ transplant recipients. ${ }^{2}$

Diarrhea is defined as an increase in frequency ( $>3$ stools per day) and volume or a decrease in consistency (loosening) of stools. Variation in reporting is high, as most of the studies follow patients' narratives to define community acquired diarrhea, which is often subjective. Stringent criteria of duration, frequency and character are often not followed. The occurrence of diarrhea in LT is frequently subjected to the immunosuppressive drugs. However, infection, concomitant drugs, existing illnesses (inflammatory bowel disease) and evolution of new entities (graft versus host reaction, lymphoproliferative disorders) are equally important.

In a study by Wong et al. diarrhea occurred in $10 \%$ of post LT patients. Leading causes were Clostridium difficile infection (CDI), cytomegalovirus (CMV)

Cite this article : Sinha R, Rahul. Post Liver Transplantation Diarrhea: Etiology, Evaluation and Treatment. Int J Med Public Health. 2021;11(3):126-32. infection and transplant medications. Ulcerative colitis was an important cause of diarrhea associated with bleeding per rectum. It carried a bad prognosis. Nearly half of the diarrheal episodes in the series occurred within first 4 months of intensive immunosuppression. ${ }^{3}$ In another study evaluating the causes of diarrhea among kidney and liver transplant patients, infection was the commonest factor (77\%). ${ }^{4}$ Any change in immunosuppression without evaluating the root cause in initial 3-4 months can increase chances of rejection. It is important to investigate a patient with diarrhea thoroughly. Risk factors associated with diarrhea in transplant patients include extremes of age (very young or very old are at increased risk), medications (antibiotics, in particular can disturb the delicate balance between commensal and pathogenic gut flora), hospital admissions (increase the chances of exposure to infective agents), work place (schools and crowded places are associated with increased transmission of infection), pets and pre-existing gastrointestinal diseases. Aim of current review is to highlight and evaluate the important causes of diarrhea following LT (Table 1).

\section{Medications}

Patients in early post LT period receive heavy dose of immunosuppression as well as antibiotic prophylaxis. The effects of drugs on GIT have been extrapolated

International Journal of Medicine and Public Health, Vol 11, Issue 3, Jul-Sep, 2021 
Table 1: Important causes of post liver transplant diarrhea

$\begin{array}{ccc}\begin{array}{c}\text { Immunosuppressants } \\ \begin{array}{c}\text { Mycophenolate mofetil } \\ \text { (most common) }\end{array}\end{array} & \begin{array}{c}\text { Infection } \\ \text { Clostridium difficile }\end{array} & \begin{array}{c}\text { Other Causes } \\ \text { Inflammatory bowel } \\ \text { disease (Ulcerative } \\ \text { colitis) }\end{array} \\ \begin{array}{c}\text { Cyclosporine } \\ \text { Sirolimus }\end{array} & \text { Cytomegalovirus } & \text { Colorectal carcinoma } \\ & \text { Rotavirus (common in } \\ \text { children) } & \text { Graft Vs Host disease } \\ \text { Azathioprine (rare) } & \begin{array}{c}\text { Parasitic infections } \\ \text { (Cryptosporidium, }\end{array} & \text { Post-transplant } \\ & \text { Cystoisospora belli, } & \text { lymphoproliferative } \\ & \text { Microsporidia) } & \text { disorder } \\ & & \text { Racterial infections } \\ & & \text { Tuberculosis, } \\ & & \text { Neuroendocrine } \\ & & \text { tumor }\end{array}$

from studies in different solid organ transplantation. None of them have exclusively focussed on the association with diarrhea.

Majority of centres use Tacrolimus or Cyclosporine (Calcineurin inhibitors - CNIs) along with tapering dose of steroids in the early period. Mycophenolate mofetil (MMF) is mainly used as an adjunct with the CNIs as maintenance therapy. The choice of regime and dosage, varies with centres, time and toxicity profile. ${ }^{5}$ Diarrhea as a side effect has been highlighted in various studies, but accurate estimation of the effect of drugs on GIT is difficult due to multiple confounding factors and drug interactions. However, some reports have demonstrated higher incidence with particular medications. In a study on LT patients, Tacrolimus was associated with nearly twice the number of diarrheal episodes in comparison to Cyclosporine. ${ }^{6}$ Similarly, MMF is also twice more toxic for GIT as compared to Azathioprine.

\section{Mycophenolate Mofetil (MMF)}

Two formulations of MMF are available for use: MMF and enteric coated mycophenolate sodium (EMP). These are prodrugs to the active component Mycophenolic acid (MPA) which is released by hydrolysis and absorbed in the gastrointestinal tract. MPA is conjugated in the liver by glucuronosyl transferase to form MPA glucuronide (inactive) and excreted in bile. It is again deconjugated (by colonic bacteria) to release MPA which enters into enterohepatic circulation. This is responsible for the second peak in plasma concentration and $10-60 \%$ of the drug activity. ${ }^{8}$ The occurrence of diarrhea with MMF is dose dependant. In a study on solid organ transplant, diarrheal episodes were twice more common with higher (twice high) serum concentration of the drug. Symptoms resolved on transiently stopping the treatment or reducing the dose. However, a reduction in dose was associated with an increase in acute rejection (28\% Vs $12 \%) .{ }^{9}$ It is prudent to maintain a delicate balance between side effects and immunosuppressive effect. Usually a dose of $2 \mathrm{~g} /$ day is better tolerated than $3 \mathrm{~g} /$ day. Route of administration is also important. Better bioavailability and early plasma peak via parenteral route may be responsible for increased diarrheal episodes as compared to oral formulae. ${ }^{10}$ Incidence of diarrhea is highest among LT recipients receiving MMF as compared to other solid organ transplant. This is true for other immunosuppresants (Tacrolimus, Azathioprine) as well. ${ }^{11}$ The incidence in LT varies from $14-15 \%$ when MMF is used alone and it increases to as high as $50 \%$ when used in combination with other drugs. ${ }^{12,13}$ Precise mechanism for the development of GI complications remains elusive. Few studies have documented villous atrophy in duodenum and mucosal erosions in distal small bowel during diarrheal episodes. Conjugated form of MPA which gets excreted in the bile may aggravate diarrhea. Another theory states that MMF induces apoptosis of enterocytes and causes dysfunction of tight junctions in between the intestinal cells. This results in secretory diarrhea. The drug is known to induce colonic ulcerations as well, hampering free water absorption. Drugs like cyclosporine compete with liver enzymes for conjugation, impede the excretion of MPA in bile and may decrease the chances of diarrhea. ${ }^{14,15}$

\section{Cyclosporine}

Diarrhea with present formulations of Cyclosporine is uncommon. Unlike Tacrolimus, it delays gastric emptying. Few reports have documented constipation as a complication associated with the use of Cyclosporine ${ }^{16}$ However, some former studies on LT have documented diarrhea in $14-47 \%$ when cyclosporine is used in combination with other drugs, but the true effect of cyclosporine on gastrointestinal functions was not clear. The mechanism of diarrhea also remains unexplained. ${ }^{17,18}$

\section{Tacrolimus}

All calcineurin inhibitors (CNIs) including Cyclosporine and Tacrolimus are metabolized by enzymes called CYP3A4 and A5, present on enterocytes. ABCB1 is the drug efflux pump which maintains an optimum concentration inside the cell. Together, the enzymes regulate the absorption of CNIs. ${ }^{19}$ Tacrolimus is a prokinetic drug and acts through motilin receptors. ${ }^{20}$ As compared to Cyclosporine containing immunosuppression regime, Tacrolimus causes more mucosal erosions (24\% Vs $18 \%$ ) as evident on endoscopy. ${ }^{21}$ Incidence of diarrhea with the use of Tacrolimus was found to be significantly high (1.5 to 2 times high) as compared to cyclosporine in various reports and randomized trials comparing different regimes following LT. ${ }^{6,22}$ Severity of gastrointestinal side effects associated with Tacrolimus is highly variable. Usually symptoms are mild and can be managed with dose reduction. Stopping immunosuppression or cross over to Cyclosporine is rarely required. Some reports in the past have shown serious GI toxicity necessitating parenteral nutrition in $6 \%$ patients. ${ }^{23}$

Diarrhea, on the other hand enhances absorption and bioavailability of Tacrolimus. Damaged enterocytes lack CYP3A system and/or ABCB1 pump. Moreover, decreased ileal transit time exposes colonic mucosa (with low CYP3A activity) to the drug. Lack of these enzymes on damaged enterocytes and colonocytes results in increased absorption of Tacrolimus. This entails intensive monitoring of the levels during diarrheal episodes to avoid toxicity. ${ }^{24,25}$

\section{Sirolimus}

Sirolimus is used following LT for hepatocellular carcinoma. It is useful in tumors within Milan's criteria due to its antiproliferative and antiangiogenic properties. ${ }^{26}$ Being a weak immunosuppressant, it is often used in combination with CNIs. On development of renal toxicity with CNIs, treatment is often shifted to Sirolimus. ${ }^{27}$

The mTOR inhibitor, like tacrolimus possesses a macrolide like structure responsible for its prokinetic effects. It downregulates the cation exchanger $\left(\mathrm{Na}^{+} / \mathrm{H}^{+}\right)$on the apical membrane of enterocytes and induces lipid malabsorption as well, thus contributing to occurrence of diarrhea in $25-42 \%$ as reported in pharmacology trials. Effect is dose dependant. However, the estimation of true evidence of diarrhea remains ambiguous as Sirolimus is often used in combination and evaluation of infection is not clearly stated in most of the studies..$^{28,29}$ The incidence reported in series on LT recipients when sirolimus was used with or without steroids ranges from $2-14 \% .30,31$ 


\section{Azathioprine}

With the use of CNIs in maintenance regime, the role of MMF or azathioprine in preventing cellular rejections has been obviated. In presence of renal dysfunction, MMF is preferred over Azathioprine as an immunosuppressant. However, in patients with HCV related transplant, Azathioprine may have an additional advantage in preventing recurrence of the disease because of its anti-HCV effect. ${ }^{32,33}$

Diarrhea is a rare complication of Azathioprine. Some patients develop severe villous atrophy which results in chronic malabsorption. ${ }^{34}$ In comparison to MMF, incidence of diarrhea with Azathioprine is quite low. ${ }^{7}$

\section{Infectious Causes of Diarrhea}

Immune-suppression is a double edged sword. Immunosuppressant are prudent to prevent acute graft rejection but at the same time increase the susceptibility to local and systemic infections. One of the most common organ systems affected is the GI tract (GIT). Rapidly dividing mucosal cells of GIT are often affected (mucosal injury and ulceration) by immunosuppression. Factors which may contribute to an increase in GI infection include: intensive care unit (ICU) stay of $>48 \mathrm{hr}$, active/ latent donor/recipient infection, anatomic or technical abnormalities, and patient's epidemiologic exposures (food and water). Infection can be bacterial (through food), viral (usually person to person or activation of latent infection) and fungal or parasitic (opportunistic). Most common manifestations include diarrhea, fever and abdominal pain.

\section{Clostridium difficile infection (CDI)}

In 1978, it was first identified as the causative agent in antibiotic-associated diarrhea and colitis. ${ }^{35}$ Liver transplant recipients receive high doses of prophylactic antibiotics in the first week. Few require additional doses of antimicrobials (plasmapheresis for $\mathrm{ABO}$ incompatible transplant, prolonged high drain output and bile leak) in the initial month. Moreover, CDI may develop denovo (without the use of antibiotics) in immune-suppressed individuals. Overall incidence of CDI ranges from 3.5-9\% among LT recipients. ${ }^{36-38}$

In a brief communication by Mittal et al. ${ }^{37}$ overall prevalence of CDI following LT was 19\%. Another retrospective study ${ }^{36}$ on 467 LT recipients documented CDI as a cause of diarrhea in $8 \%$ and majority of the events were reported in the first month. Another study ${ }^{38}$ recorded CDI in 14\% patients over a period of 1.8 years. More than $40 \%$ occurred within 1 week. Symptoms include: watery non-bloody diarrhea, abdominal cramps, fever and nausea. Symptoms can vary from mild disease to a life-threatening fulminant form in 5\% (toxic megacolon).

Diagnosis of CDI is established by a positive laboratory test for C. difficile and/or its toxins. Typical "horse barn" smell heralds the possibility of CDI. Stool of a suspected patient is cultured on a selective agar plate called CCFA (cycloserine cefoxitin fructose agar) and incubated in an anaerobic atmosphere for $48 \mathrm{hr}^{39}$

Stool culture done on CCFA has high sensitivity (>90\%) and specificity $(>98 \%)$ but does not differentiate between toxic and non-toxic strains. Presence of toxin can be established by tissue culture, enzyme immune assay, counter immunoelectrophoresis, dot immunobinding assay, latex agglutination test, rapid membrane test and polymerase chain reaction. A multistep algorithm is followed to establish the diagnosis: Glutamate Dehydrogenase (GDH) plus toxin or Nucleic acid amplification test (NAAT) plus toxin. ${ }^{39}$ Patients with confirmed CDI should be isolated. Stringent measures for infection control (hand washing, decontamination and antibiotics) should be implemented. Updated guidelines for the treatment of Clostridium difficile infection is as follows: ${ }^{40,41}$
- First episode is treated with oral vancomycin (125 mg 4 times a day for 10 days) or Fidaxomicin (200 mg twice daily for 10 days).

- Metronidazole (500 mg per oral thrice daily for 10 days) is used when vancomycin/fidaxomicin is unavailable or contraindicated.

- Fulminant infection (hypotension/shock/megacolon) requires high dose of vancomycin (500mg 4 times daily). In the presence of ileus, drug is administered per rectally along with metronidazole.

- Recurrent infection is treated with tapered and pulsed regimen of vancomycin or fidaxomicin. However, multiple recurrences $(>2)$ entail the use of fecal microbiota transplantation (nasogastric route or rectal route or oral frozen fecal capsules) with $>90$ percent response rate.

Bezlotoxumab, a monoclonal antibody against Toxin $\mathrm{B}$ has been recently approved for prevention of recurrence in refractory CDI. ${ }^{42}$

\section{Cytomegalovirus}

Cytomegalovirus (CMV) is the commonest viral infection post LT. It most commonly involves the GIT, but can affect lungs and allograft as well. Infection can be localized or organ invasive. A special property of CMV is viral latency. It persists in a dormant stage inside the host cells and gets activated under the effect of immunosuppression. ${ }^{43}$ Inhibition of T-lymphocyte proliferation by CNIs is the predisposing factor. In a study by Sakr et al. ${ }^{44}$ Cyclosporine was more commonly (27\%) associated with CMV enteritis than Tacrolimus (20\%). Mycophenolate mofetil also adds to the risk of CMV reactivation, necessitating dose reduction in $6-10 \%$ patients. Overall incidence in LT is $18-29 \%,{ }^{45,46}$ but it affects nearly two-third of the recipients with a donor-recipient mismatch (Donor positive and recipient negative, $\mathrm{D}+/ \mathrm{R}-$ ). It usually manifests in the phase of intensive immunosuppression, i.e., first 3-6 months after LT. This justifies the use of CMV prophylaxis (Ganciclovir or Valganciclovir) for the first 3 months, especially in presence of serology mismatch. In study on LT recipients, use of ganciclovir significantly reduced the incidence of CMV in D+/R- subgroup. ${ }^{46}$ However, disease may occur even 6 months after stopping the prophylaxis and it is more likely to be tissue-invasive. Apart from serology mismatch (D+/R-), other risk factors include: use of lymphocyte depleting agents (thymoglobulin, high dose MMF), re-transplantation and allograft rejection. It can affect the whole GIT. Initial symptoms include nausea, vomiting, diarrhea and leucopenia. Endoscopy in early phase usually reveals non-specific features: erythematous mucosa and shallow ulcers. Inclusion bodies may be seen on biopsy. If the disease is not recognized and treated early, it becomes tissue invasive and may cause lung infection, bleeding and/or intestinal perforation. CMV reactivation up-regulates $\mathrm{T}$ cells and may induce allograft rejection (Vanishing Duct Syndrome). It can invade vascular endothelium and result in hepatic artery thrombosis as well. It also increases the risk of other opportunistic infections including bacterial, viral and fungal diseases. ${ }^{47,48}$ Detection of CMV (antigen) in blood or stool by Polymerase chain reaction (PCR) or DNA hybridization techniques is useful for diagnosis. Drug of choice for an established CMV infection is intravenous Ganciclovir or oral Valganciclovir or Foscarnet, till the viral antigenemia is cleared. ${ }^{49}$

\section{Rotavirus}

Rotavirus infection is a common cause for diarrhea among pediatric LT recipients ( $<3$ years). Incidence can be as high as $50 \%$. However, it is rarely observed among adult recipients $(<2 \%) .{ }^{50}$ The virus induces epithelial cell death in small intestine. Colonic mucosa is less affected. Resultant depletion in the brush border enzymes (lactase) leads to acquired lactose intolerance. ${ }^{51}$ Symptoms include acute watery diarrhea, fever, lower abdominal pain and generalized weakness. Dehydration and dyselectrolytemia often necessitate readmission or prolongation of 
post-operative stay. ${ }^{50}$ Poor hygiene is considered the most important predisposing factor as the usual mode of infection is feco-oral transmission (contaminated food or water). ${ }^{52}$ Diagnosis can be easily established by Immunochromatographic card test or toxin detection by ELISA in patient's stool sample. Management includes supportive care with intravenous fluid replacement and reduction in the dose of immunosuppression. Disease is often self-limiting (lasts 4-10 days). Severity of infection can be potentially reduced by pre-transplant vaccination. ${ }^{53}$

Cryptosporidium, Cystoisospora belli and Microsporidia: These parasitic infections often result in chronic diarrhea among immunocompromised individuals and organ transplant recipients. Infection is transmitted via feco-oral route, primarily through contaminated water. ${ }^{54}$ Patients usually complain of persistent watery diarrhea, bloating and abdominal pain. Diagnosis of these microorganisms is established by direct fecal smear examination using special stains (Modified Acidfast stain, Weber trichrome stain, and fluorochrome stain) to identify the oocysts. ${ }^{55}$ Antigen based PCRs of stool samples are also useful. Thiabendazole and ivermectin has been found to be effective against the parasites.

Bacterial infections: Intestinal infection by bacteria in LT recipients is common. Apart from CDI (discussed earlier); Yersinia enterocolitica, shigella, Escherichia coli and Clostridium can also be transmitted through contaminated food. ${ }^{56}$ It is prudent to maintain a good hygiene and practice healthy food habits in the initial years of transplant to avoid such infections. The treatment protocol is similar to that in immunocompetent individuals.

Intestinal microbiome plays a key role in gut-liver symbiosis and immune homeostasis. The microbial composition of gut flora is significantly altered (abundance of Enterobacteriaceae and Enterococcus) in end stage liver disease (ESLD). Moreover, intestinal mucosal damage due to portal hypertensive gastropathy and enteropathy is accountable for bacterial translocations and development of various complications (encephalopathy, bacterial peritonitis, sepsis, organ failures, and malignant changes) in the pre-transplant period. As reported by Kato et al..$^{57}$ composition and diversity of gut microbiota post liver transplant correlates with the grade of preoperative liver decompensation (high model for end stage liver disease or Child Pugh scores). In 4-8 weeks, as the need for antibiotics and immune-suppressants decreases, diversity is restored and incidence of diarrhea decreases. Delay in restoration or a decrease in biodiversity is a predictor of blood stream infection and acute cellular rejection. Advances in culture independent characterization of complex intestinal microbiome (metagenomics and metabolomics) and their clinical implications on post- transplant outcomes (microbiomics) are potential areas of research for microbial-based therapy in liver transplantation. ${ }^{58}$

\section{Ulcerative colitis}

Inflammatory bowel disease (IBD) is a rare cause of diarrhea post LT. Annual incidence of de novo IBD following LT is $\sim 0.2 \%$. However, it may develop in $30 \%$ of patients transplanted for autoimmune hepatitis or Primary sclerosing cholangitis (PSC) over a period of 10 years. Preexisting disease tends to recur in $70 \%$ patients. Chances of a flare up (severe disease) are uncommon as the patient is immune-suppressed (steroids and azathioprine). A good number of patients (0-82\%) experience an initial improvement in symptoms. ${ }^{59}$ They usually reappear on tapering of steroids. Patients who are started on 5-aminosalicyalic acid early after LT (before the symptoms appear), have decreased risk of recurrence. ${ }^{60,61}$ In a large series exclusively on LT for PSC and autoimmune hepatitis, authors documented increased requirement of medications for IBD during the first year post $\mathrm{LT}^{60}$ Use of tacrolimus in initial period is the probable reason for frequent flare ups. The CNI induces down regulation of T-cell by inducing apoptosis. T-cells play a crucial role in maintaining immunological homeostasis in gut. Moreover, absorption of Tacrolimus is enhanced in IBD due to increased permeability. Cyclosporine on the other hand is associated with decreased risk of recurrence of IBD. Addition of Azathioprine to the regime further reduces the chances of flare up. Disease activity is usually controlled on medications and seldom requires surgical intervention. ${ }^{60,61}$

\section{Colorectal Carcinoma}

Diarrhea in post LT patients as a manifestation of colorectal carcinoma (CRC) is a rare encounter (1\%). Incidence of CRC post LT is no different from the general population. However, relaxation in age criteria for LT and improved survival in these patients increases the risk of developing malignancies including CRC. Patients being operated for PSC related liver disease with history of IBD are particularly at high risk (4 fold) of developing bowel cancer, more frequently involving the right colon. ${ }^{62}$ Diagnosis can be readily established by endoscopy and biopsy. Contrast enhanced cross sectional imaging helps in accurate staging and planning of treatment. Curative colectomies are safe even in transplanted patients. Experience in perioperative chemotherapy in this cohort is limited. However, oxaliplatin and 5-fluorouracil are well tolerated. ${ }^{62}$ Some authors have suggested prophylactic colectomy during or after LT to improve survival in presence of risk factors: long term IBD $>10$ years, pancolitis or severe dysplasia or presence of colonic polyps in patients $>40$ years at transplantation. ${ }^{62,63}$ High risk candidates who do not undergo colectomy should be put on intensive surveillance protocol (annual endoscopy) to pick early changes. ${ }^{64}$

\section{Graft Vs Host Disease (GVHD)}

GVHD is a life threatening condition. It is the reaction of donor lymphocytes against host tissue antigens. It commonly follows a bone marrow transplant ( $50 \%)$. Incidence following LT is low $(0.1-2 \%)$. It affects organs with high turnover of cells - skin, mucosa of GIT and bone marrow. The most common symptoms include skin rash (80$90 \%)$, fever (60\%) and diarrhea (50-55\%). Pancytopenia may develop in $30-70 \%$ patients. It usually presents at the end of first month following LT (2-8 weeks). ${ }^{65,66}$ Common risk factors include: complete human leukocyte antigen match, recipients $>65$ years, young donors, recipientdonor age difference $>20$ years, pre-LT blood transfusion, autoimmune hepatitis, re-transplantation and viral infections (CMV) ${ }^{66,67}$ GVHD is fatal in $>80 \%$ even at the best of centres. Early recognition is the key to successful management. Initial symptoms include diarrhea and skin rash. High index of suspicion is necessary as the symptoms overlap with more common disorders like drug reaction, bacterial and viral infection. Histopathology of the skin (epidermal vacuolization, lymphocytic exocytosis) or GIT mucosa (crypt apoptosis) is often useful. Treatment includes escalation of immunosuppression and addition of prophylactic broad spectrum antibiotics, antifungals and antivirals. Use of steroid pulse alone is associated with high mortality (85\%). Addition of IL-2 antagonists or TNF-alpha inhibitors has shown promising results $(\sim 50 \%$ survival). ${ }^{66}$

\section{Post-transplant Lymphoproliferative Disorder (PTLD)}

PTLD is a rare but important and potentially treatable cause of chronic diarrhea. It may present within 20 days to several years post LT. ${ }^{68,69}$ Disease manifestation within 1 year is termed as early onset PTLD. It is commonly a B-cell type lymphoma associated with Epstein-Barr Virus (EBV). Another form which manifests 3-5 years post LT is usually EBV negative and more aggressive. ${ }^{70}$ Risk factors include: age (incidence is more following LT in pediatric population - $10 \%$ Vs $1-3 \%$ in adults), 
dose of immunosuppression (risk increases with increase in dose as in acute rejection), type of drugs used (CNIs have the highest risk, while mTOR inhibitors - Rapamycin (Sirolimus) has anti-proliferative effect on in-vitro PTLD derived cell lines) and EBV status of recipient and donor (highest risk in donor positive and recipient negative for EBV). Mumtaz et al. ${ }^{68}$ in their series of $>1300$ LT recipients, reported the incidence as $2.3 \%$. Median interval between LT and development of PTLD was 60 months. Commonest etiology was LT for autoimmune causes $(47 \%)$ followed by HCV. The reason stated was higher dose of immunosuppression required to prevent rejection in autoimmune diseases. Nearly $50 \%$ of patients with PTLD in the series received treatment (escalation of immunosuppression) for acute rejection.

Symptoms in PTLD vary with the organ involved. GIT is affected in one-fourth to half the cases. Most common symptoms include: gastrointestinal bleeding $(>95 \%)$, chronic diarrhea with protein losing enteropathy (90\%), fever, and weight loss $(90 \%){ }^{71}$ Poor prognostic factors include advanced Ann Arbor scale, poor functional status and extra-nodal disease. Diagnosis in presence of GIT involvement can be established by endoscopy (colonoscopy/gastroduodenocopy) or image guided (computed tomography) deep mucosal biopsy and bone marrow biopsy after ruling out infectious causes. Typical findings of a lymphoma confirmed on immunohistochemistry with positive in situ hybridization for EBV RNA confirms the diagnosis. Consensus on the most suitable treatment protocol for PTLD is lacking. However, there is enough evidence to suggest cut on immunosuppression initially, especially the CNIs. Some authors have suggested the use of sirolimus due to its potent anti-proliferative effect. ${ }^{72,73}$ This is followed by chemotherapy: CHOP (Cyclophoshamide, Hydroxydaunorubicin, Oncovin and Prednisolone) with or without Rituximab. Some reports have shown remarkable response to Rituximab alone. $\mathrm{CHOP}$ is usually administered every 3 weeks for 6 cycles. Rituximab is administered weekly for 4 weeks. Patients with anatomically localized disease or with significant symptoms (bowel obstruction or bleeding) are subjected to surgical resections. Successful treatment entails good long term survival $(\sim 60 \%$ at 5 years following diagnosis). ${ }^{68,73}$

\section{Allergic Diarrhea}

Allergic diarrhea is not uncommon in liver transplant recipients. Passive transfer of allergen sensitivity from donors to adult recipients is an established mechanism. Milk products, sea foods, eggs, and peanut are common culprits. Development of denovo food allergies has also been documented and is attributed to various factors: large organ size (increased probability of transferring sensitized lymphocytes), type of immunosuppression (Tacrolimus enhances $\operatorname{IgE}$ production and increases intestinal permeability), recipients' age (children are more susceptible as have immature immune regulatory response), and family history of atopy. Symptoms (pain, diarrhea, bloating, vomiting) usually resolve by elimination of the offending food allergen or switching (immunosuppression) from tacrolimus to cyclosporine. ${ }^{74,75}$

\section{Rare Causes of Diarrhea}

Few causes of diarrhea post LT described in literature are limited to case reports and small case series. Tuberculosis is sometimes seen to flare up following LT, and is usually extra pulmonary. Some may present with diarrhea. Presence of granulomas in liver explants is an important risk factor. Isoniazid prophylaxis in such cases prevents development of the disease and mortality associated with it. ${ }^{76}$

One report from China documented vasoactive intestinal peptide as a cause of diarrhea in one of the recipients 17 months after a split liver transplant. It was confirmed with subsequent rise in Chromogranin

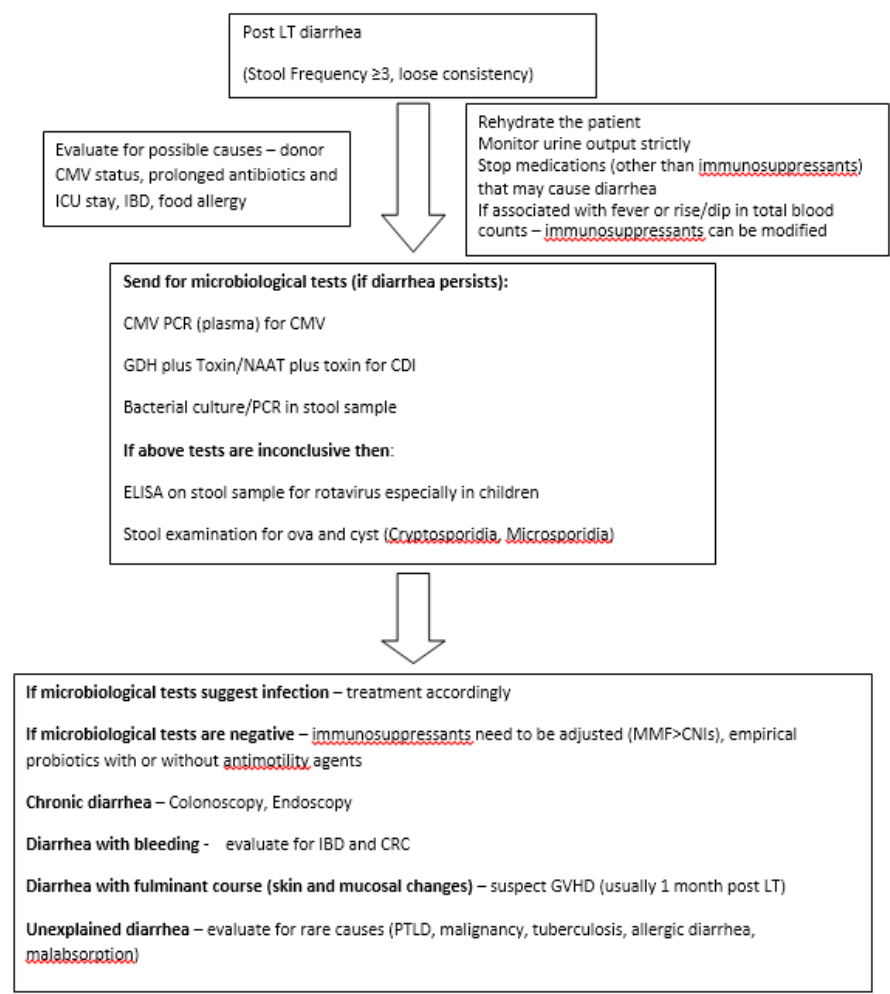

Figure 1: Management algorithm for diarrhea post Liver transplantation.CDI - Clostridium difficile infection; CMV - Cytomegalovirus; CNI - Calcineurin inhibitors; CRC - colorectal carcinoma; ELISA - Enzyme linked immunoassay; GDH Glutamate Dehydrogenase; GVHD - graft versus host disease; IBD - Inflammatory Bowel Disease; ICU - intensive care unit; MMF - Mycophenolate mofetil; LT - Liver Transplant; NAAT - Nucleic acid amplification test; PCR - Polymerase chain reaction; PTLD - Post-transplant lympho-proliferative Disorder.

level. Cross-sectional imaging demonstrated a lesion in the transplanted lobe, which was effectively treated by radiofrequency ablation. ${ }^{77}$

\section{Prevention of diarrhea}

The most effective way to decrease the incidence of diarrhea is frequent hand washing (patient as well as the care providers). Crowded places should be avoided in first few months following transplant. Street foods, undercooked or stale food, unpeeled raw fruits and vegetables should be avoided. Drinking water should be purified before use.

\section{CONCLUSION}

Pathogenesis of diarrhea following LT is complex. Often diarrheal episodes are self-limiting. If symptoms persist, it is prudent to establish the etiology (Figure 1). Analysis of time gap, risk factors, previous episodes, medications and dietary changes aid in the process of establishing the diagnosis. Infection remains an important cause in early post-transplant period, especially when patient is discharged from the hospital. High index of suspicion is required to suspect and establish the less frequent but potentially treatable causes like PTLD, IBD and GVHD. Incidence of diarrhea associated with an immunosuppressant is highly variable, and in few trials similar to or slightly more than a placebo. ${ }^{12,78}$ Usual strategy to reduce the dose or stop the immunosuppressant (especially MMF) as the first step in unexplained diarrhea is questionable. Loss of graft is more life threatening than diarrhea itself. Administration of appropriate antimicrobials (antivirals/antibacterials) and changes in life style (food habits, hygiene) should be contemplated before altering transplant medications. Prospective studies to particularly evaluate the 
causes of diarrhea and management, comprehensive documentation and reporting of adverse events will help in devising an appropriate plan of management for post LT diarrhea.

\section{ACKNOWLEDGEMENT}

We thank Dr. Hunaid Hatimi who rendered support and expertise in quick reviews and critical analysis.

\section{CONFLICT OF INTEREST}

The authors declare that there is no conflict of interest.

\section{ABBREVIATIONS}

CD: Clostridium difficile; CDI: Clostridium difficile infection; CHOP: Cyclophoshamide, Hydroxydaunorubicin, Oncovin and Prednisolone; CMV: Cytomegalovirus; CNI: Calcineurin inhibitors; CRC: colorectal carcinoma; EBV: Epstein Barr Virus; ELISA: Enzyme linked immunoassay; EMP: mycophenolate sodium; GDH: Glutamate Dehydrogenase; GI: gastrointestinal; GIT: gastrointestinal tract; GVHD: graft versus host disease; HCV: Hepatitis C Virus; IBD: Inflammatory Bowel Disease; ICU: intensive care unit; LT: liver transplant; MMF: Mycophenolate mofetil; MPA: Mycophenolic acid; mTOR: mammalian target of rapamycin; NAAT: Nucleic acid amplification test; PCR: Polymerase chain reaction; PSC: Primary sclerosing cholangitis; PTLD: Post-transplant Lymphoproliferative Disorder.

\section{REFERENCES}

1. Rubin $\mathrm{RH}$. Gastrointestinal infectious disease complications following transplantation and their differentiation from immunosuppressant-induced gastrointestinal toxicities. Clin Transplant. 2001;15:11-22.

2. Ginsburg PM, Thuluvath PJ. Diarrhea in liver transplant recipients: Etiology and management. Liver Transpl. 2005;11(8):881-90.

3. Wong NA, Bathgate AJ, Bellamy CO. Colorectal disease in liver allograft recipients - a clinicopathological study with follow-up. Eur J Gastroenterol Hepatol. 2002;14(3):231-6.

4. Bunnapradist S, Neri L, Wong W, et al. Incidence and risk factors for diarrhea following kidney transplantation and association with graft loss and mortality. Am J Kidney Dis. 2008;51(3):478-86.

5. Kirk AD. Induction immunosuppression. Transplantation. 2006;82(5):593-602.

6. U.S. Multicenter FK506 Liver Study Group. A comparison of tacrolimus (FK 506) and cyclosporine for immunosuppression in liver transplantation. N Engl J Med. 1994;331:1110-5

7. Germani G, Pleguezuelo M, Villamil F, et al. Azathioprine in liver transplantation: A reevaluation of its use and a comparison with mycophenolate mofetil. Am J Transplant. 2009;9(8):1725-31.

8. Colom H, Lloberas N, Andreu F, et al. Pharmacokinetic modeling of enterohepatic circulation of mycophenolic acid in renal transplant recipients. Kidney Int 2014;85(6):1434-43.

9. Shaw LM, Kaplan B, Denofrio D, et al. Pharmacokinetics and concentrationcontrol investigations of mycophenolic acid in adult transplant patients. Ther Drug Monit. 2000;22(1):14-9.

10. Pescovitz MD, Conti D, Dunn J, et al. Intravenous mycophenolate mofetil: Safety, tolerability, and pharmacokinetics. Clin Transplant. 2000;14(3):179-88.

11. Pescovitz MD, Navarro MT. Immunosuppressive therapy and post-transplantation diarrhea. Clin Transplant. 2001;15:23-8.

12. Wiesner R, Rabkin J, Klintmalm G, et al. A randomized double-blind comparative study of mycophenolate mofetil and azathioprine in combination with cyclosporine and corticosteroids in primary liver transplant recipients. Liver Transpl. $2001 ; 7(5): 442-50$.

13. Fairbanks KD, Thuluvath PJ. Mycophenolate mofetil monotherapy in liver transplant recipients: A single center experience. Liver Transpl. 2004;10(9):1189-94.

14. Kamar N, Faure P, Dupuis E, et al. Villous atrophy induced by mycophenolate mofetil in renal-transplant patients. Transpl Int. 2004;17(8):463-7.

15. Maes BD, Dalle I, Geboes K, et al. Erosive enterocolitis in mycophenolate mofetil-treated renal-transplant recipients with persistent afebrile diarrhea Transplantation. 2003;75(5):665-72.

16. Maes BD, Vanwalleghem J, Kuypers D, et al. Differences in gastric motor activity in renal transplant recipients treated with FK-506 versus cyclosporine Transplantation. 1999;68(10):1482-5

17. Malinowski M, Martus P, Lock JF, et al. Systemic influence of immunosuppres- sive drugs on small and large bowel transport and barrier function. Transpl Int. $2011 ; 24(2): 184-93$.

18. European FK506 Multicentre Liver Study Group. Randomised trial comparing tacrolimus (FK506) and cyclosporin in prevention of liver allograft rejection. Lancet. 1994;344(8920):423-8.

19. Staatz CE, Goodman LK, Tett SE. Effect of CYP3A and ABCB1 single nucleotide polymorphisms on the pharmacokinetics and pharmacodynamics of calcineurin inhibitors: Part I. Clin Pharmacokinet. 2010:49(3):141-75.

20. Naesens $M$, Verbeke $K$, Vanrenterghem $Y$, et al. Effects of gastric emptying on oral mycophenolic acid pharmacokinetics in stable renal allograft recipients. $\mathrm{Br}$ J Clin Pharmacol. 2007;63(5):541-7.

21. Telkes G, Peter A, Tulassay Z, et al. High frequency of ulcers, not associated with Helicobacter pylori, in the stomach in the first year after kidney transplantation. Nephrol Dial Transplant. 2011;26(2):727-32.

22. Levy G, Villamil F, Samuel D, et al. Results of list, a multicenter, randomized study comparing cyclosporine microemulsion with C2 monitoring and tacrolimus with CO monitoring in de novo liver transplantation. Transplantation. $2004 ; 77(11): 1632-8$.

23. Fisher A, Schwartz M, Mor E, et al. Gastrointestinal toxicity associated with FK 506 in liver transplant recipients. Transplant Proc. 1994;26(6):3106-7.

24. Nakamura A, Amada N, Haga I, et al. Effects of elevated tacrolimus trough levels in association with infectious enteritis on graft function in renal transplant recipients. Transplant Proc. 2014;46(2):592-4.

25. Thorn M, Finnstrom N, Lundgren S, et al. Cytochromes P450 and MDR1 mRNA expression along the human gastrointestinal tract. Br J Clin Pharmacol. 2005;60(1):54-60

26. Geissler EK, Schnitzbauer AA, Zülke C, et al. Sirolimus Use in Liver Transplant Recipients with Hepatocellular Carcinoma: A Randomized, Multicenter, OpenLabel Phase 3 Trial. Transplantation. 2016;100(1):116-25.

27. Asrani SK, Leise MD, West $C P$, et al. Use of sirolimus in liver transplant recipients with renal insufficiency: A systematic review and meta-analysis. Hepatology. 2010;52(4):1360-70

28. Vasquez EM. Sirolimus: A new agent for prevention of renal allograft rejection. Am J Health Syst Pharm. 2000;57(5):437-51

29. Yang J, Zhao X, Patel $A$, et al. Rapamycin Inhibition of mTOR Reduces Levels of the $\mathrm{Na}+/ \mathrm{H}+$ Exchanger 3 in Intestines of Mice and Humans, Leading to Diarrhea. Gastroenterology. 2015;149(1):151-62.

30. Fairbanks KD, Eustace JA, Fine D, et al. Renal function improves in liver transplant recipients when switched from a calcineurin inhibitor to sirolimus. Liver Transpl. 2003;9(10):1079-85

31. Neff GW, Montalbano M, Slapak-Green G, et al. A retrospective review of sirolimus (Rapamune) therapy in orthotopic liver transplant recipients diagnosed with chronic rejection. Liver Transpl. 2003;9(5):477-83.

32. Fischer L, Sterneck M, Gahlemann CG, et al. A prospective study comparing safety and efficacy of mycophenolate mofetil versus azathioprine in primary liver transplant recipients. Transplant Proc. 2000;32(7):2125-7.

33. Zekry A, Gleeson M, Guney $\mathrm{S}$, et al. A prospective cross-over study comparing the effect of mycophenolate versus azathioprine on allograft function and viral load in liver transplant recipients with recurrent chronic HCV infection. Liver Transpl. 2004;10(1):52-7.

34. Weclawiak H, Ould-Mohamed A, Bournet B, et al. Duodenal villous atrophy A cause of chronic diarrhea after solid-organ transplantation. Am J Transplant. 2011;11(3):575-82

35. Bartlett JG, Moon N, Chang TW, et al. Role of Clostridium difficile in antibioticassociated pseudomembranous colitis. Gastroenterology. 1978;75(5):778-82.

36. Albright JB, Bonatti $\mathrm{H}$, Mendez J, et al. Early and late onset Clostridium difficile associated colitis following liver transplantation. Transpl Int. 2007;20(10):85666 .

37. Mittal C, Hassan S, Arshad S, et al. Clostridium difficile infection in liver transplant recipients: A retrospective study of rates, risk factors and outcomes. Am J Transplant. 2014;14(8):1901-7.

38. Dallal RM, Harbrecht BG, Boujoukas AJ, et al. Fulminant Clostridium difficile: An underappreciated and increasing cause of death and complications. Ann Surg. 2002;235(3):363-72

39. McDonald LC, Gerding DN, Johnson S, et al. Clinical practice guidelines for Clostridium difficile infection in adults and children: 2017 update by the Infectious Diseases Society of America (IDSA) and Society for Healthcare Epidemiology of America (SHEA). Clin Infect Dis. 2018;66(7):987-94

40. Burnham CA, Carroll KC. Diagnosis of Clostridium difficile infection: An ongoing conundrum for clinicians and for clinical laboratories. Clin Microbiol Rev. 2013;26(3):604-30.

41. $\mathrm{Ng} \mathrm{KE}$. Updates in the management of Clostridium difficile for adults. US Pharm. 2019;44(4):HS-9-12.

42. Alonso CD, Mahoney MV. Bezlotoxumab for the prevention Clostridium difficile Infection: A review of current evidence and safety profile. Infect Drug Resist. $2019 ; 12: 1-9$

43. Razonable RR, Emery VC. $11^{\text {th }}$ Annual Meeting of the IHMF (International Herpes Management Forum). Management of CMV infection and disease in trans- 
plant patients. 27-29 February 2004. Herpes. 2004;11(3):77-86.

44. Sakr M, Hassanein T, Gavaler J, et al. Cytomegalovirus infection of the upper gastrointestinal tract following liver transplantation--incidence, location, and severity in cyclosporine- and FK506-treated patients. Transplantation. 1992;53(4):786-91.

45. Singh N, Wannstedt $C$, Keyes $L$, et al. Who among cytomegalovirus-seropositive liver transplant recipients is at risk for cytomegalovirus infection?. Liver Transpl. 2005;11(6):700-4

46. Paya C, Humar A, Dominguez E, et al. Efficacy and safety of valganciclovir vs oral ganciclovir for prevention of cytomegalovirus disease in solid organ transplant recipients. Am J Transplant. 2004;4(4):611-20.

47. Madalosso C, Souza DNFJ, Ilstrup DM, et al. Cytomegalovirus and its association with hepatic artery thrombosis after liver transplantation. Transplantation. 1998;66(3):294-7.

48. Bruminhent J, Razonable RR. Management of cytomegalovirus infection and disease in liver transplant recipients. World J Hepatol. 2014;6(6):370-83.

49. Tan BH. Cytomegalovirus Treatment. Curr Treat Options Infect Dis. 2014;6(3):256-70.

50. Stelzmueller I, Wiesmayr S, Swenson BR, et al. Rotavirus enteritis in solid organ transplant recipients: an underestimated problem?. Transpl Infect Dis. 2007;9(4):281-5

51. Kanwar SS, Singh V Vinayak VK, et al. Differential tropism of EB rotavirus (serotype 3 ) to small intestine of homologous murine model. Acta Virol. 1994;38(5):269-76

52. Parashar UD, Gibson CJ, Bresee JS, et al. Rotavirus and severe childhood diarrhea. Emerg Infect Dis. 2006;12(2):304-6.

53. Bresee JS, Parashar UD, Widdowson MA, et al. Update on rotavirus vaccines. Pediatr Infect Dis J. 2005;24(11):947-52.

54. Agholi M, Hatam GR, Motazedian MH. Microsporidia and coccidia as causes of persistence diarrhea among liver transplant children: Incidence rate and species/genotypes. Pediatr Infect Dis J. 2013;32(2):185-7.

55. Cama VA, Mathison BA. Infections by Intestinal Coccidia and Giardia duodenalis. Clin Lab Med. 2015;35(2):423-44.

56. Hernandez MDP, Martin P, Simkins J. Infectious Complications after LiverTransplantation. Gastroenterol Hepatol. 2015;11(11):741-53.

57. Kato K, Nagao M, Miyamoto K, et al. Longitudinal analysis of the intestinal microbiota in liver transplantation. Transplantation Direct. 2017;3(4):e144.

58. Kriss M, Verna EC, Rosen HR, Lozupone CA. Functional microbiomics in liver transplantation: Identifying novel targets for improving allograft outcomes. Transplantation. 2019;103(4):668-78.

59. Verdonk RC, Dijkstra G, Haagsma EB, et al. Inflammatory bowel disease afte liver transplantation: Risk factors for recurrence and de novo disease. Am J Transplant. 2006;6(6):1422-9.

60. Riley TR, Schoen RE, Lee RG, et al. A case series of transplant recipients who despite immunosuppression developed inflammatory bowel disease. Am J Gastroenterol. 1997;92(2):279-82.

61. Feagan BG. Maintenance therapy for inflammatory bowel disease. Am J Gastroenterol. 2003;98(12):S6-17.

62. Vera A, Gunson BK, Ussatoff V, et al. Colorectal cancer in patients with inflammatory bowel disease after liver transplantation for primary sclerosing cholangi- tis. Transplantation. 2003;75(12):1983-8.

63. Nishihori T, Strazzabosco M, Saif MW. Incidence and management of colorectal cancer in liver transplant recipients. Clin Colorectal Cancer. 2008;7(4):260-6.

64. Eaden JA, Mayberry JF. British Society for Gastroenterology; Association of Coloproctology for Great Britain and Ireland. Guidelines for screening and surveillance of asymptomatic colorectal cancer in patients with inflammatory bowe disease. Gut. 2002;51:V10-12.

65. Akbulut S, Yilmaz M, Yilmaz S. Graft-versus-host disease after liver transplantation: A comprehensive literature review. World J Gastroenterol. 2012;18(37):5240-8.

66. Murali AR, Chandra S, Stewart Z, et al. Graft Versus Host Disease After Liver Transplantation in Adults: A Case series, Review of Literature, and an Approach to Management. Transplantation. 2016;100(12):2661-70.

67. Chan EY, Larson AM, Gernsheimer TB, et al. Recipient and donor factors influence the incidence of graft-vs.-host disease in liver transplant patients. Liver Transpl. 2007;13(4):516-22.

68. Mumtaz K, Faisal N, Marquez M, et al. Post-transplant lymphoproliferative disorder in liver recipients: Characteristics, management, and outcome from single-centre experience with $>1000$ liver transplantations. Can J Gastroenterol Hepatol. 2015;29(8):417-22

69. Dotti G, Fiocchi R, Motta $T$, et al. Lymphomas occurring late after solid organ transplantation - Influence of treatment on the clinical outcome. Transplantation. 2002;74(8):1095-102

70. Dierickx D, Cardinaels N. Posttransplant lymphoproliferative disorders following liver transplantation: Where are we now?. World J Gastroenterol. 2015;21(39):11034-43.

71. Younes BS, Ament ME, McDiarmid SV, et al. The involvement of the gastrointestinal tract in posttransplant lymphoproliferative disease in pediatric liver transplantation. J Pediatr Gastroenterol Nutr. 1999:28(4):380-5.

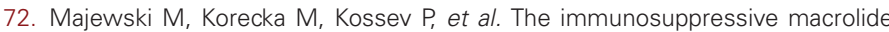
RAD inhibits growth of human Epstein-Barr virus-transformed B lymphocytes in vitro and in vivo: A potential approach to prevention and treatment of posttransplant lymphoproliferative disorders. Proc Natl Acad Sci U S A. 2000;97(8):428590.

73. Jain A, Mazariegos G, Kashyap R, et al. Pediatric liver transplantation. A single center experience spanning 20 years. Transplantation. 2002;73(6):941-7.

74. Maarof G, Krzysiek R, Décline JL, Cohen J, Habes D, Jacquemin E. Management of post-liver transplant-associated IgE-mediated food allergy in children. $J$ Allergy Clin Immunol. 2011;127(5):1296-8.

75. Balogun R, Carr TF, Desai AP, Jie T, Pan JJ. Transfer of peanut allergy from dono to recipient after liver transplant. Annals of Hepatology 2019;18(3):508-13.

76. Olithselvan A, Rajagopala S, Vij M, et al. Tuberculosis in liver transplant recipients: Experience of a South Indian liver transplant center. Liver Transpl. 2014;20(8):960-6.

77. Haiqing W, Jiayin $Y$, Jian $Y$, et al. Intractable and dramatic diarrhea in liver transplantation recipient with vasoactive intestinal peptide-producing tumor after split liver transplantation: A case report. Transplant Proc. 2015:47(1):171-3.

78. Mathew TH. A blinded, long-term, randomized multicenter study of mycophenolate mofetil in cadaveric renal transplantation: results at three years. Tricontinental Mycophenolate Mofetil Renal Transplantation Study Group. Transplantation. 1998;65(11):1450-4.

Cite this article : Sinha R, Rahul. Post Liver Transplantation Diarrhea: Etiology, Evaluation and Treatment. Int J Med Public Health. 2021;11(3):126-32. 